\title{
Impedance Eduction in a Duct Using the Linearized Euler Equations
}

\author{
Willie R. Watson* and Michael G. Jones ${ }^{\dagger}$ \\ NASA Langley Research Center, Hampton VA, 23681-2199
}

\begin{abstract}
An impedance eduction technique that is based upon a numerical solution to the linearized Euler equations coupled with the minimization of an objective function is presented. The linearized Euler equations are solved numerically using a cubic finite element method and the minimum of the objective function is obtained via a gradient based optimizer. The mean flow is allowed to have a gradient in two coordinate directions and the impedance of the liner may vary arbitrarily over the liner surface. A new regularized wall impedance boundary condition presented by Rienstra which contains the effects of the mean boundary layer thickness is implemented. Impedances are educed for a perforate over honeycomb liner using test data acquired in the Langley Grazing Flow Impedance Tube. The impedance of the liner is educed both with an upstream source and a downstream source. The method is validated by comparing the educed impedance to a benchmark method. The primary conclusion of the study is that the impedances educed for upstream and downstream sources are not well matched and that the primary effect of the boundary thickness is to reduce the resistance of the liner.
\end{abstract}

\section{Nomenclature}

$\begin{array}{ll}{\left[A^{g}\right],[A]} & =\text { global stiffness matrices for the computational domain } \\ {\left[A_{u}\right],\left[B_{u}\right],\left[C_{u}\right]} & =\text { upper wall matrices containing the unknown admittance coefficients } \\ c_{0}, \rho_{0}, M_{0}(z, x) & =\text { sound speed, mean static density, axial component of mean flow Mach number } \\ \{E(z, x)\}, E_{u}(z) & =\text { field error vector for the linearized Euler equations, upper wall error function } \\ F_{I}(z, x) & =\text {-D cubic Hermite polynomial basis functions for finite element analysis } \\ \{F\},\{\Phi\} & =\text { vector containing source effects, vector containing the unknown nodal coefficients } \\ f, \omega & =\text { sound source frequency in Hertz, circular frequency in radian per second }(2 \pi f) \\ H, L, W & =\text { height of duct, length of duct, width of duct } \\ i & =\text { unit imaginary number }(\sqrt{-1}) \\ k & =\text { free space wave number }\left(\omega / c_{0}\right) \\ L_{1}, L_{2} & =\text { axial location of the leading and trailing edge of the test liner, respectively } \\ M, N & =\text { number of unevenly spaced grid lines in the } x \text { and } z \text { directions, respectively } \\ p(z, x) & =\text { acoustic pressure field } \\ u(z, x), v(z, x) & =\text { axial and vertical component of the acoustic particle velocity vector, respectively } \\ p_{I}, u_{I}, v_{I}, \mathcal{M}_{I} & =\text { nodal degrees of freedom for } p(z, x), u(z, x), v(z, x), \text { and } \mathcal{M}_{0}(z, x), \text { respectively } \\ R(z), \chi(z) & =\text { normalized acoustic resistance function, normalized acoustic reactance function } \\ z, x, y, t & =\text { axial coordinate, vertical coordinate, horizontal coordinate, and time, respectively } \\ \beta(z), \zeta(z) & =\text { normalized acoustic admittance function, normalized acoustic impedance function } \\ \beta_{1}, \frac{d \beta_{1}}{d z} & =\text { nodal admittance coefficients for local node } 4 \text { in a typical upper wall finite element } \\ \beta_{2}, \frac{d \beta_{2}}{d z} & =\text { nodal admittance coefficients for local node } 3 \text { in a typical upper wall finite element } \\ \sigma, \Psi(\zeta) & =\text { boundary layer thickness, objective function whose minimum occur at the liner impedance }\end{array}$

Subscripts:

$I, J=$ basis function counter $(I=1,2,3, \ldots 16)$, microphone counter $(J=1,2,3 \ldots 79)$

$q \quad=$ basis function counter for the upper wall boundary $(q=9,10,13,14)$

FEM, Meas = a finite element computed quantity, a measured quantity

$e, s \quad=$ an exit plane quantity, a source plane quantity

${ }^{*}$ Distinguished Research Associate, Computational AeroSciences Branch, Liner Physics Group, Associate Fellow AIAA.

${ }^{\dagger}$ Senior Research Scientist, Structural Acoustics Branch, Liner Physics Group, Associate Fellow AIAA. 


\section{Introduction}

TCREASINGLY stringent national and international noise constraints have resulted in continued emphasis on im-

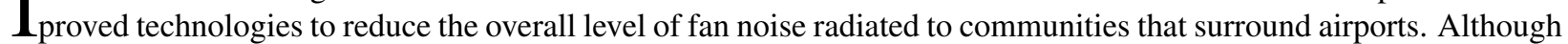
locally-reacting, sound absorbing structures (acoustic liners) mounted in the aircraft engine nacelles currently provide significant fan-noise reduction, further optimization is required to increase their noise reduction capacity and bandwidth. The critical intrinsic parameter used in this optimization is the locally-reacting acoustic impedance of the liner. Therefore, an accurate knowledge of this quantity is critical for the design of quieter aircraft. The acoustic impedance of a liner in the presence of grazing flow is defined as the ratio of the acoustic pressure to the normal component of acoustic particle velocity at the surface of the liner. Simultaneous measurement of both quantities (i.e., the acoustic pressure and the normal component of acoustic particle velocity) at the surface of the liner is problematic at best. Instead, techniques have been developed to evaluate the impedance of a liner by using a mixture of numerical methods and acoustic pressure measurements taken away from the liner surface. These techniques are referred to as impedance eduction techniques in the duct acoustics literature, and may be further classified as either direct or indirect methods.

In direct methods, the axial propagation constant of a single mode in the duct is typically measured using Pronylike methods ${ }^{1-4}$ and the wall impedance boundary condition is used to extract the liner impedance without the need to implement troublesome iterative methods. Although direct methods are extremely simple to apply, they lack generality to model arbitrary duct geometries, arbitrary mean flows, and variable impedance liners. Indirect methods (which rely on iterative methods) do not suffer from these limitations.

For 30 years the NASA Langley Research Center has invested a significant effort in the development of indirect impedance eduction tools and experimental rigs for the evaluation of acoustic liner impedance. ${ }^{5-10}$ These tools are directly applicable to rectangular geometries for which the test liner is mounted in one wall of the duct while the remaining walls remain rigid. They have the additional restriction that the sound field between the two rigid walls adjacent to the test liner consists of only the plane wave mode. When applied to the Langley facilities, these impedance eduction tools are applicable to uniform test liners installed in the NASA Langley Grazing Flow Impedance Tube (GFIT). ${ }^{6}$ Further, nearly all of the impedance eduction results presented in the literature from Langley facilities have focused on uniform impedance liners, the uniform mean flow assumption, the use of the Ingard-Myers boundary condition ${ }^{11}$ coupled with the convected Helmholtz equation (CHE), and the application of indirect impedance eduction methods. Some results have been presented using shear flow but these results have been restricted to a direct method that is based on the solution of the one-dimensional Pridmore Brown equation. ${ }^{6}$

The objective of this paper is to develop an indirect impedance eduction method that can accommodate either uniform or sheared flow profiles. Unlike other direct impedance eduction methods, the sheared flow profile may contain boundary layer growth and the surface impedance may vary with position along the surface. The governing equations that are solved to educe the impedance are the linearized Euler equations (LEE). The Ingard-Myers boundary condition (used with the uniform flow assumption in earlier papers) is replaced with the regularized wall impedance boundary condition developed by Rienstra. ${ }^{12}$ In uniform flow, the new regularized boundary condition is expected to produce more accurate results than that of Ingard-Myers because it incorporates the effects of a finite boundary layer thickness. Data are acquired in the NASA Langley GFIT for a conventional liner. This liner consists of a perforated plate bonded to a honeycomb core, and is typical of liner structures currently installed in aircraft engine nacelles for the reduction of engine fan noise.

Results are first presented for a liner with known impedance with data synthesized from an exact mode solution under the assumption of uniform grazing flow. This exact data is used to test the integrity of the LEE method. Next, impedances are educed using measured data from the GFIT and these results are compared to that of the extensively tested CHE method. ${ }^{5}$ Results are presented for both an upstream propagating source and a downstream propagating source. Finally, the LEE method is used to evaluate the effects of the boundary layer thickness on the educed impedance under the assumption of a uniform grazing flow for both upstream and downstream propagating sound sources.

This paper is organized as follows. The measurement apparatus is described in the following section (Section II). Section III presents the governing differential equations, the wall impedance boundary conditions, and inflow-outflow boundary conditions that are used to educe the liner impedance. Section IV gives the details of the numerical method that are used to solve for the acoustic field in the duct. Section V describes the impedance eduction technique that computes the impedance spectra from the measured data. The details of the construction of the test liner are given in Section VI. Selected results are given in Section VII and the relevant conclusions of the paper are given in Section VIII. 


\section{The Measurement Apparatus}

Measured data needed to perform the current study are acquired in the NASA Langley GFIT. A sketch of this stateof-art research facility is shown in Fig. 1. The GFIT has a cross-sectional geometry of $50.8 \mathrm{~mm} \times 63.5 \mathrm{~mm}$, such that

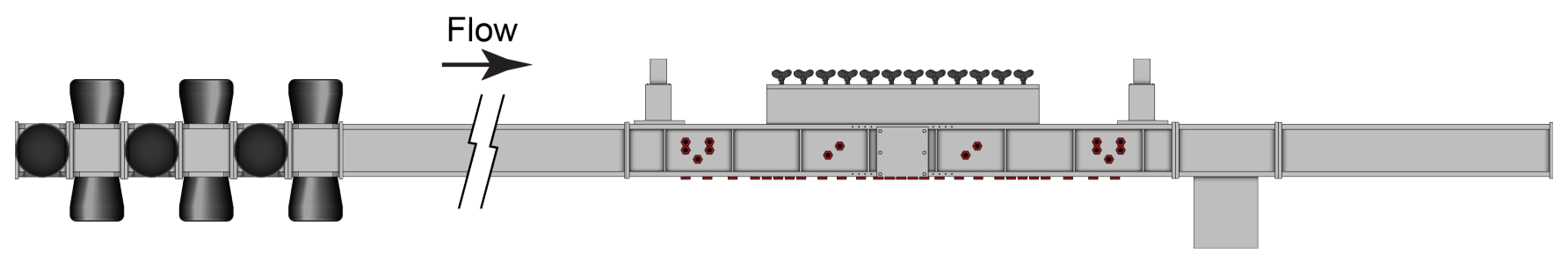

Figure 1. Sketch of the LaRC grazing flow impedance tube (GFIT) and instrumentation.

higher-order modes in the horizontal and vertical dimensions cut on at different frequencies. It allows convenient evaluation of acoustic liners with lengths up to $609.6 \mathrm{~mm}$. Mean flow is from left to right in the figure. High-pressure air is supplied upstream and a vacuum blower is located on the downstream end, such that the test window containing the acoustic liner is exposed to near-ambient pressure conditions for mean flows up to Mach 0.6. The surface of the test liner forms a portion of the upper wall of the flow duct. As depicted in Fig. 1, the source section consists of twelve acoustic drivers mounted upstream (exhaust mode) of the liner to generate tones (one frequency at a time) for sound pressure levels up to $150 \mathrm{~dB}$ over a frequency range of 0.4 to $3.0 \mathrm{kHz}$. Although not shown in Fig. 1, six acoustic drivers are located downstream of the liner. The downstream drivers are used to generate sound waves that propagate against the flow.

Figure 2 shows a sketch of the rectangular flow duct and coordinate system for which impedance eduction analysis

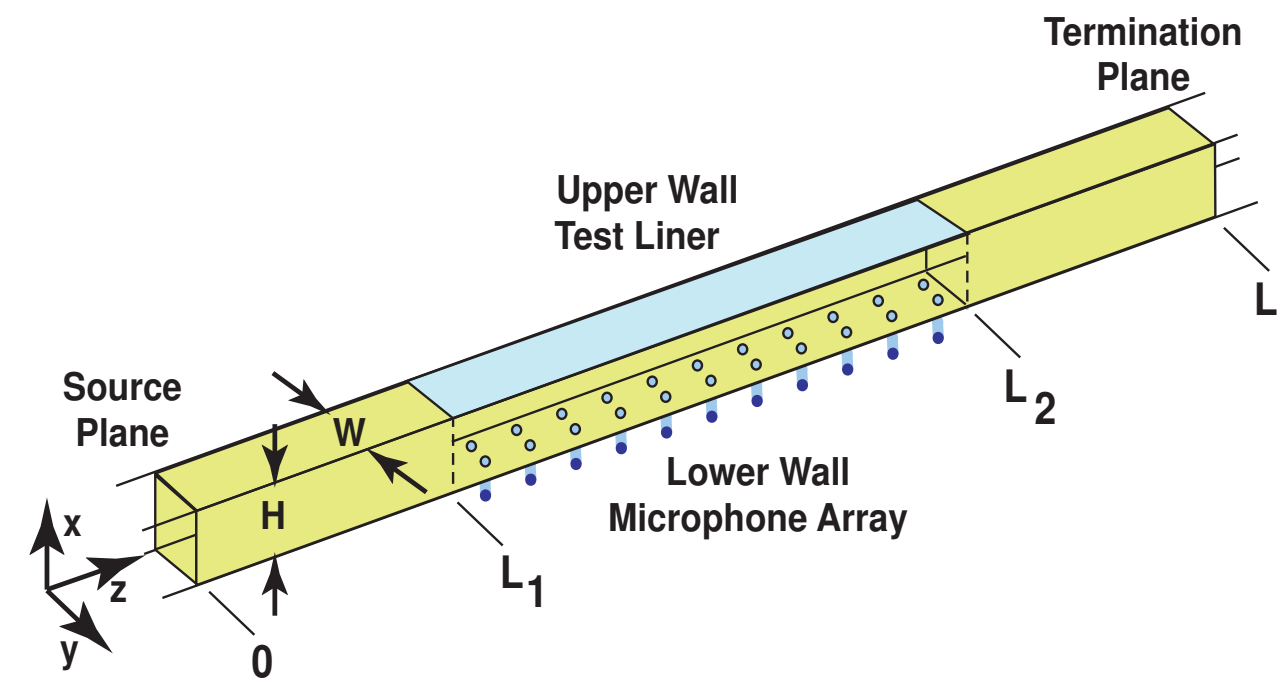

Figure 2. Schematic of the LaRC grazing flow impedance tube (GFIT) flow duct.

is performed. The origin of the coordinate system is located upstream of the liner in the lower left corner of the duct. The flow duct has a height, $H$, of $63.5 \mathrm{~mm}$, a width, $W$, of $50.8 \mathrm{~mm}$, and a length $L$, of $1016 \mathrm{~mm}$. As shown in the figure, the surface of the test liner forms a portion of the upper wall for $L_{1} \leq z \leq L_{2}$ and the remainder of the duct consists of rigid walls. The test liner has an unknown, normalized impedance that is denoted by $\zeta(z)$. Throughout this paper, all impedances are normalized with the characteristic impedance, $\rho_{0} c_{0}$, of the air flowing in the duct. Fifty-three microphones flush-mounted in the lower wall (opposite the test liner) are used to measure the acoustic pressure field over the axial extent $0 \leq z \leq L$ (see Fig. 2) of the liner. These fifty-three lower wall microphones are located at the axial locations $z=z_{J}(J=1,2, \ldots 53)$ on the lower wall. The impedance eduction method uses these measured acoustic wall pressures to educe the impedance of the test liner. To further improve the accuracy of the eduction, the current study will utilize sixteen additional microphones in the impedance eduction. Eight of the microphones are located in 
the hard wall section upstream of the liner and eight microphones are located in the hard wall section downstream of the liner. These additional microphones are flush-mounted in the upper and two side walls of these hard wall sections. This brings the total number of microphones used in the impedance eduction to seventy-nine.

\section{Governing Differential Equations and Boundary Conditions}

This paper focuses attention to the flow duct depicted in Fig. 2 for which the boundary layer grows and the steady flow velocity vector is purely axial. Because the duct is of constant cross-sectional area, the mean density and sound speed are assumed constant. However, the mean axial velocity is assumed a function of both the axial and vertical coordinate (i.e., $z$ and $x$, respectively). The assumption of a region with constant density and sound speed at some finite distance downstream of the duct entrance is a reasonable approximation in the NASA Langley Grazing Flow Impedance Tube (GFIT). The purpose of this section of the paper is to present the governing differential equations, the wall impedance boundary conditions, and the inflow-outflow boundary conditions that are used for the impedance eduction.

\section{A. Governing Differential Equations}

The linearized equations for continuity of mass, axial momentum, and vertical momentum can be written as three equations in three unknown variable $(p, u, v)$ :

$$
\{E\}=\left\{\begin{array}{l}
\left(i k+\frac{\partial M_{0}}{\partial z}\right) p+M_{0} \frac{\partial p}{\partial z}+\rho_{0} c_{0}\left(\frac{\partial u}{\partial z}+\frac{\partial v}{\partial x}\right) \\
M_{0} \frac{\partial M_{0}}{\partial z} p+\frac{\partial p}{\partial z}+\rho_{0} c_{0}\left(i k u+M_{0} \frac{\partial u}{\partial z}+\frac{\partial M_{0}}{\partial z} u\right)+\rho_{0} c_{0} \frac{\partial M_{0}}{\partial x} v \\
\frac{\partial p}{\partial x}+\rho_{0} c_{0}\left(i k v+M_{0} \frac{\partial v}{\partial z}\right)
\end{array}\right\}=\left\{\begin{array}{l}
0 \\
0 \\
0
\end{array}\right\}
$$

In Eq. (1), the free space wavenumber, $k$, is assumed sufficiently low that high order horizontal modes are cut off. Under this assumption, the sound field is independent of the coordinate $y$ (see Fig. 1) and depends only on position in the $(z, x)$ plane. The LEE method of impedance eduction presented in the next section is based on the solution to Eq. (1).

\section{B. Wall Boundary Conditions}

The lower wall of the flow duct depicted in Fig 2 is fully rigid. Thus, the lower wall boundary condition must require that the normal component of acoustic particle velocity vanish at the lower wall:

$$
v(z, 0)=0
$$

The upper wall of the duct is partially lined with a sound absorbing material that contains wall cavities. Because the effects of viscosity on the sound waves in the wall cavities have been neglected in Eq. (1), it is convenient to model the sound absorbing properties of the upper wall via a wall impedance boundary condition. The authors used the Rienstra $^{12}$ regularized wall impedance boundary condition for the lined portion of the upper wall:

$$
\rho_{0} c_{0}\left[1-\frac{\sigma}{3}\left(\frac{\partial}{\partial x}+\frac{2 i k}{\zeta}+\frac{M_{0}}{\zeta} \frac{\partial}{\partial z}\right)\right] v(z, H)=\frac{1}{\zeta}\left[1+\frac{1}{i k}\left(M_{0} \frac{\partial}{\partial z}-\sigma \frac{\partial^{2}}{\partial z^{2}}\right)\right] p(z, H)
$$

Note that the lining material is assumed locally reacting so that the upper wall boundary condition (Eqs. (3)) cannot be used if the sound absorbing material is nonlocally reacting (i.e., the acoustic material is a bulk absorber). Equation (3) is used as the wall boundary condition in this paper for the following reasons:

1. It is convenient for modeling the rigid wall portion of the upper wall. This is achieved by setting $\sigma$ to zero (i.e., $\sigma=0)$ and the wall impedance to an infinite value (i.e., $1 / \zeta(z)=0$ ).

2. It reduces to the Ingard-Myers ${ }^{11}$ boundary condition that is used in our previous papers when the boundary layer thickness is assumed infinitely thin (i.e., when $\sigma=0$ ):

$$
\rho_{0} c_{0} v(z, H)=\left(1+\frac{M_{0}}{i k} \frac{\partial}{\partial z}\right)\left(\frac{p(z, H)}{\zeta}\right)
$$


3. It is expected to be more accurate than the Ingard-Myers boundary condition because it accounts for the effects of a small but finite boundary layer thickness, $\sigma$.

4. For realistic shear flows (i.e., $\sigma=0$ and $M_{0}(z, H)=0$ ) it reduces to the commonly used no-slip boundary condition:

$$
v(z, H)=\frac{p(z, H)}{\rho_{0} c_{0} \zeta}
$$

\section{Inflow-Outflow Boundary Conditions}

Free boundaries, such as those at the inflow (i.e., $z=0$ ) and outflow (i.e., $z=L$ ) boundaries, across which matter passes freely are generally difficult to analyze. These boundaries do not arise from a natural physical situation (such as a wall impedance) so that suitable forms of the boundary conditions required on them are not obvious. It is necessary that some of these boundary conditions model a specified physical situation, such as the duct termination or source of noise. Other conditions should be consistent with the compatibility equations of the linearized Euler system (see Eqs. (1)) in order that the problem will be well-posed. Inflow-outflow boundary conditions are generally analyzed using the method of characteristics. ${ }^{13}$

A characteristic analysis of the Linearized Euler equations in two space dimensions shows that two boundary conditions are required at the inflow boundary (i.e., there are two incoming characteristics at the inflow boundary). Further, only one boundary condition is required at the outflow boundary (i.e., only one characteristic is incoming at the outflow boundary). At the inflow boundary, we specify the inflow acoustic pressure, $p_{s}(x)$, and the normal component of the inflow acoustic particle velocity, $v_{s}(x)$, as the inflow boundary conditions:

$$
p(0, x)=p_{s}(x), \quad v(0, x)=v_{s}(x)
$$

At the outflow boundary, we specify the outflow acoustic pressure, $p_{e}(x)$, as the outflow boundary condition:

$$
p(L, x)=p_{e}(x)
$$

Both the inflow boundary data (i.e., $p_{s}(x)$, and $v_{s}(x)$ ) and the outflow boundary data (i.e., $p_{e}(x)$ ) are measured via mode synthesis. The mode synthesis is performed using upstream and downstream flush mounted microphone arrays, located in the hard wall sections upstream and downstream of the liner.

\section{The Numerical Method}

Equations (1)-(5) form a well-posed boundary value problem. Although exact solutions are not available for arbitrary boundary layer thicknesses, $\sigma$, Mach number profiles, $M_{0}(z, x)$, and wall impedance functions, $\zeta(z)$, these equations can be solved numerically. The numerical method chosen to solve these equations is the finite element method. Only enough detail is presented in this section to highlight the major differences between the CHE method presented in an earlier paper ${ }^{5}$ and the LEE method presented in this paper. Those interested in a more elementary treatment of the finite element method may consult the excellent text by Chandrakant and Abel. ${ }^{14}$ When applied to the current acoustic problem, the finite-element method may be interpreted as approximations to the acoustic pressure and acoustic particle velocity field. This is achieved by dividing the duct into $N$ grid lines in the axial $(z)$ and $M$ grid lines in the vertical $(x)$ directions. Unlike in past works, ${ }^{5}$ the grid lines are unequally spaced to allow for the effects of mean flow gradients in both the vertical and axial directions, and to accommodate the effects of unequally spaced microphones in the hard wall section of duct upstream and downstream of the test liner. A typical rectangular element is formed by axial grid lines located at $z=z_{I}$ and $z=z_{I+1}$ and by vertical grid lines located at $x=x_{J}$ and $x=x_{J+1}$ as shown in Fig. 3. Each element, $e$, consists of four local node numbers, and the objective is to obtain the unknown acoustic pressure and particle velocity fields at the nodes of each finite element.

Within each finite element, the acoustic variables $p, u$, and $v$, as well as the mean flow Mach number, $M_{0}$, are expanded as a series of basis functions, $F_{I}$, and nodal degrees of freedom $p_{I}, u_{I}, v_{I}$, and $\mathcal{M}_{I}$

$$
p=\sum_{I=1}^{16} F_{I}(z, x) p_{I}, \quad u=\sum_{I=1}^{16} F_{I}(z, x) u_{I}, \quad v=\sum_{I=1}^{16} F_{I}(z, x) v_{I}, M_{0}=\sum_{I=1}^{16} F_{I}(z, x) \mathcal{M}_{I},
$$

Here, the first four coefficients in the series expansion for the acoustic pressure variable (i.e., $p_{1}, p_{2}, p_{3}, p_{4}$ ) correspond to nodal degrees of freedom at local node 1 (see Fig. 3), the second four coefficients (i.e., $p_{5}, p_{6}, p_{7}, p_{8}$ ) correspond to 


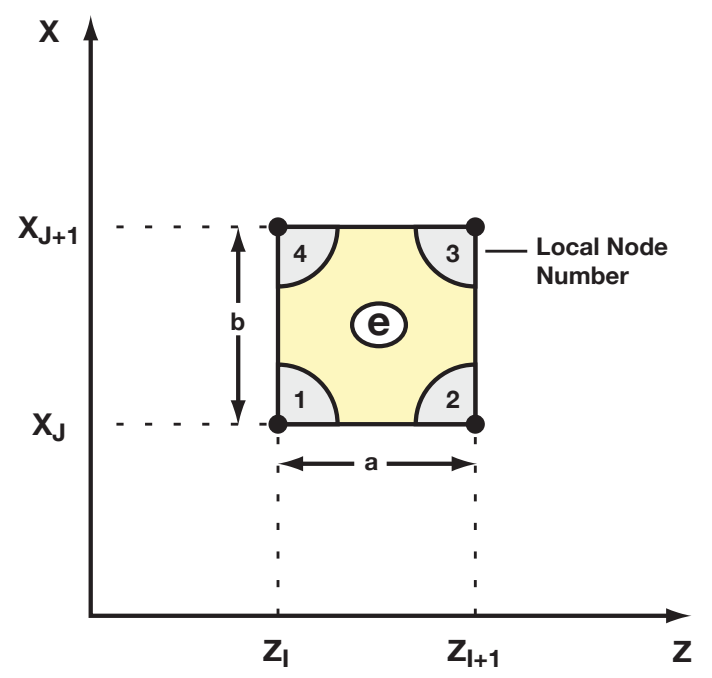

Figure 3. A typical finite-element with width $a$ and height $b$.

nodal degrees of freedom at local node 2 , and so forth and so on. The coefficients in the series expansion for $u$ (i.e., $u_{I}$ ) and $v$ (i.e., $v_{I}$ ) have a similar meaning. In selecting the series expression in Eq. (6), the completeness and compatibility requirements for convergence of the finite element method to the exact solution of Eq. (1) are the most important considerations. The sixteen basis functions, $F_{I}(z, x)$, are 2D cubic Hermite polynomials that are written explicitly in standard finite-element texts ${ }^{14}$ and are not presented in this paper. Each series in Eq. (6) is constructed such that each acoustic variable, (i.e., $p(z, x), u(z, x)$, or $v(z, x)$ ) and its normal derivative are continuous across interelement boundaries. This implies, for example, that for the acoustic pressure variable, that $p, \frac{\partial p}{\partial z}, \frac{\partial p}{\partial x}$, and $\frac{\partial^{2} p}{\partial z \partial x}$ are the required nodal degrees of freedom at each of the four nodes of the element. Identical nodal degrees of freedom are used for $u$ and $v$ with the acoustic pressure variable replaced with the appropriate acoustic particle velocity variable.

To account for the effects of nonuniform lining impedance, the reciprocal of the variable normalized impedance function (i.e., $\beta(z)$ ) is represented as a cubic polynomial along each of the $N-1$ upper wall elements

$$
\frac{1}{\zeta(z)}=\beta(z)=F_{13}(z, b) \beta_{1}+F_{14}(z, b) \frac{d \beta_{1}}{d z}+F_{9}(z, b) \beta_{2}+F_{10}(z, b) \frac{d \beta_{2}}{d z}
$$

Note that for the $N-1$ columns of unequally spaced upper wall elements, $4(N-1)$ admittance coefficients are to be determined. Substituting the series expressions in Eq. (6) into each of the $(N-1)(M-1)$ finite elements, requiring that the field error vector, $\{E\}$, be orthogonal to each basis function, $F_{I}(z, x)$, and assembling the elements in the usual manner ${ }^{14}$ leads to a global matrix equation of the form:

$$
\left[A^{g}\right]\{\Phi\}=\{0\}
$$

Here, $\{\Phi\}$, is a column vector of length $12 N M$ that contains the nodal coefficients $\left(p_{I}, u_{I}\right.$, and $\left.v_{I}\right)$ for the $N M$ grid points and $\left[A^{g}\right]$ is the $12 N M \times 12 N M$ complex, asymmetric, block tridiagonal, stiffness matrix for the computational domain. It is easily shown that there are exactly $(3 N-2)$ tridiagonal blocks in $\left[A^{g}\right]$ and each tridiagonal block has an order of $12 M$.

Implementation of the Rienstra wall impedance boundary condition into the cubic finite analysis is a unique contribution of this paper. To satisfy the upper wall boundary condition, the upper wall error function, $E_{\mathrm{u}}(z)$, where:

$$
E_{\mathrm{u}}(z)=\rho_{0} c_{0}\left[1-\frac{\sigma}{3}\left(\frac{\partial}{\partial x}+\frac{2 i k}{\zeta}+\frac{M_{0}}{\zeta} \frac{\partial}{\partial z}\right)\right] v(z, H)-\frac{1}{\zeta}\left[1+\frac{1}{i k}\left(M_{0} \frac{\partial}{\partial z}-\sigma \frac{\partial^{2}}{\partial z^{2}}\right)\right] p(z, H)
$$


is minimized by making it orthogonal to the basis functions, $F_{q}(z, b)$, where $q=9,10,13,14$. Note that the other twelve basis functions are identically zero when evaluated along the upper wall and are not used in the minimization of $E_{\mathrm{u}}(z)$. Contributions to the minimization of the upper wall error function for each upper wall element are assembled in the usual manner, ${ }^{14}$ to obtain the global matrix equation for the upper wall boundary condition:

$$
\left[A_{u}\right]\left\{V_{u}\right\}+\left[B_{u}\right] \frac{\partial}{\partial x}\left\{V_{u}\right\}=\left[C_{u}\right]\left\{P_{u}\right\}
$$

In Eq. (10), $\left[A_{u}\right],\left[B_{u}\right]$ and $\left[C_{u}\right]$ are each $2 N \times 2 N$ block tridiagonal matrices that contain the unknown wall admittance coefficients (i.e., $\beta_{1}, \frac{d \beta_{1}}{d z}, \beta_{2}, \frac{d \beta_{2}}{d z}$ ) for each of the $(N-1)$ wall elements. $\left\{V_{u}\right\}, \frac{\partial}{\partial x}\left\{V_{u}\right\}$ and $\left\{P_{u}\right\}$ are $2 N \times 1$ column vectors. $\left\{V_{u}\right\}$ contains the vertical acoustic particle velocity, $v$, and its axial derivative, $\frac{\partial v}{\partial z}$, at the $N$ upper wall boundary nodes. Similarly, $\left\{P_{u}\right\}$, contains the acoustic pressure, $p$, and its axial derivative, $\frac{\partial p}{\partial z}$, at the $N$ upper wall boundary nodes. Finally, $\frac{\partial}{\partial x}\left\{V_{u}\right\}$ is the axial derivative of $\{V\}$ and contains $\frac{\partial v}{\partial x}$ and $\frac{\partial^{2} v}{\partial x \partial z}$ at the $N$ upper wall boundary nodes.

Implementing the upper wall boundary condition (see Eq. (10)) as multipoint constraints on Eq. (8), then constraining the nodal degrees of freedom in the series expansions for $p$ and $v$ to satisfy the lower wall boundary condition (see Eq. (2)), the inflow boundary condition (see Eq. (4)), and the outflow boundary condition (see Eq. (5)), leads to a large system of sparse, asymmetric, matrix equations of the form:

$$
[A]\{\Phi\}=\{F\}
$$

The matrix $[A]$ is the complex, asymmetric stiffness matrix, whose order is $12 N M$, and $\{F\}$ is a $12 N M \times 1$ column vector that contains the effects of the inflow and outflow boundary conditions. The solution to Eq. (11) is obtained using a parallel, sparse, equation solver with equation reordering to minimize fill-ins.

\section{Impedance Eduction Technique}

The solution to Eq. (11) gives a solution for acoustic pressure, $p(z, x)$, as a function of the undetermined admittance coefficients. These coefficients are determined from the fifty-three microphones located on the lower wall and the sixteen additional microphones located in the rigid wall sections of duct upstream and downstream of the liner. The procedure is to determine values of these admittance coefficients, such that the finite element solution obtained from Eq. (11) reproduces the measured acoustic pressure at the seventy-nine microphones to within some acceptable tolerance. This is obtained by minimizing the objective function:

$$
\psi(\zeta)=\sum_{J=1}^{I=79}\left\|p_{\text {Meas }}\left(z_{J}, x_{J}\right)-p_{\mathrm{FEM}}\left(z_{J}, x_{J}\right)\right\|
$$

Note that this objective function may be interpreted as the difference between the measured acoustic pressure field, $p_{\text {Meas }}\left(z_{J}, x_{J}\right)$, and that computed by the finite-element method, $p_{\mathrm{FEM}}\left(z_{J}, x_{J}\right)$. Because the optimization algorithm makes use of the objective function gradient to find its minimum, and this function is available only in numerical form (i.e., as a solution of Eq. (11)), Stewart's adaptation of the Davidon-Fletcher-Powell (SDFP) optimization algorithm ${ }^{15}$ is used to obtain the minimum in this work.

\section{Description of the Test Liner}

A schematic of the test liner is shown in Fig. 4. The liner is a conventional liner and is composed of a punched aluminum facesheet bonded to an aluminum honeycomb core that is in turn bonded to a rigid backplate. The face sheet is $0.635 \mathrm{~mm}$ thick with holes that are $0.991 \mathrm{~mm}$ in diameter. The percent open area ratio is 15 percent. The honeycomb core has a cell size of $9.525 \mathrm{~mm}$ and a depth of $38.100 \mathrm{~mm}$. This liner was chosen for two primary reasons. First, it is typical of a production liner and its impedance is known to show some sensitivity to the mean flow Mach number. Second, its liner impedance has previously been educed with the well tested CHE method. ${ }^{5}$ Thus, the CHE method results are used as a baseline for comparing LEE results. 


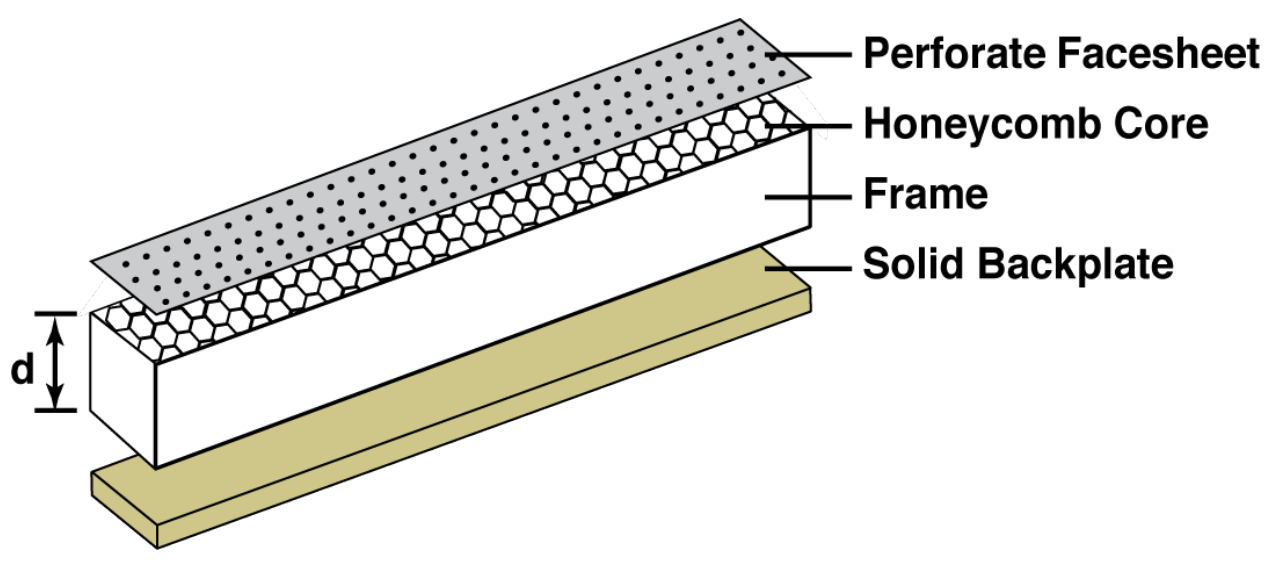

Figure 4. Schematic of conventional liner.

\section{Results and Discussion}

This section presents results for the LEE method of impedance eduction. Its accuracy is assessed by comparing educed impedance to the well tested CHE method (whenever possible). Examples are presented for 1) data synthesized from an exact mode solution, and 2) data acquired in the GFIT with the conventional liner installed. Each exercise makes the assumption of a uniform mean flow. As the test liner is known to have a uniform structure, we reduce the computational cost by allowing each upper wall element to have a uniform wall impedance. This is achieved by choosing the four admittance coefficients in each wall element to have identical values:

$$
\beta_{1}=\beta_{2}=\frac{1}{R+i \chi}, \quad \frac{d \beta_{1}}{d z}=\frac{d \beta_{2}}{d z}=0
$$

Thus, the design variables are the uniform dimensionless resistance, $R$, and the uniform dimensionless reactance, $\chi$, of the wall lining (i.e., $\zeta=R+i \chi$ ). The computational grid for all impedance eductions is a uniform grid with eighty-one grid lines in the axial direction $(N=81)$ and twenty-six grid lines in the vertical direction $(M=26)$. This grid is more than sufficient to resolve all cuton modes propagating in the duct for source frequencies less than $2.8 \mathrm{kHz}$. It is worth noting that LEE results presented in this section were run on eight CPUs, and the wall clock time to educe the impedance for a typical frequency was less than one minute.

\section{A. Impedance Eduction Using Synthesized Data}

In the first example, the LEE method is tested by utilizing data synthesized from an exact mode solution for outgoing waves in an infinitely long duct. The synthesized data results afford one the opportunity to test the LEE impedance eduction methodology when the data are free from measurement uncertainties and boundary layer effects. An exact mode solution is valid when the upper wall lining is uniform and infinite in extent (i.e., there are no hard wall sections of the duct) and the mean flow Mach number, $M_{0}$, is constant. Under these conditions, the exact solution in the duct is easily obtained using mode theory

$$
\begin{gathered}
p(z, x)=\sum_{n=0}^{\infty} A_{n} e^{-i K_{n} z} \cos \left(\lambda_{n} x\right), \quad v(z, x)=\sum_{n=0}^{\infty} \frac{A_{n} \lambda_{n}}{i \rho_{0} c_{0}\left[k-M_{0} K_{n}\right]} e^{-i K_{n} z} \sin \left(\lambda_{n} x\right) \\
\left(\lambda_{n}\right)^{2}=k^{2}-\left(1-M_{0}\right)^{2}\left(K_{n}\right)^{2}-2 k M K_{n} \\
i k H\left(1-\frac{M_{0} K_{n}}{k}\right)^{2}-\zeta\left(\lambda_{n} H\right) \tan \left(\lambda_{n} H\right)=0
\end{gathered}
$$

where $A_{n}$ is the right moving wave coefficient. Note that when the uniform upper wall impedance $(\zeta)$ is known, the axial propagation constants (i.e., $K_{n}$ ) can be obtained from the solution to the exact transcendental equation (Eq. 16), and the duct eigenvalue $\left(\lambda_{n}\right)$ can be extracted from the dispersion relation (Eq. (15)).

The assumed resistance and reactance spectra given in Table 1 are used to construct the least attenuated mode 
Table 1. Normalized resistance and reactance of test liner

\begin{tabular}{ccccc}
\hline \hline & \multicolumn{2}{c}{$M_{0}=0.0$} & \multicolumn{2}{c}{$M_{0}=0.257$} \\
$\mathrm{f}$ & $\mathrm{R}$ & $\chi$ & $R$ & $\chi$ \\
\hline \hline 0.4 & 0.24 & -3.77 & 0.89 & -4.86 \\
0.6 & 0.18 & -2.19 & 1.26 & -1.90 \\
0.8 & 0.15 & -1.54 & 1.10 & -1.35 \\
1.0 & 0.12 & -1.00 & 0.83 & -1.20 \\
1.2 & 0.16 & -0.58 & 0.89 & -0.66 \\
1.4 & 0.19 & -0.18 & 0.94 & -0.47 \\
1.6 & 0.16 & 0.04 & 0.85 & -0.25 \\
1.8 & 0.14 & 0.32 & 0.81 & -0.05 \\
2.0 & 0.21 & 0.64 & 0.92 & 0.14 \\
2.2 & 0.21 & 0.88 & 0.86 & 0.29 \\
2.4 & 0.26 & 1.10 & 0.92 & 0.52 \\
2.6 & 0.57 & 1.59 & 0.94 & -0.14 \\
2.8 & 0.94 & 1.43 & 1.29 & -0.73 \\
3.0 & 1.43 & 1.20 & 0.37 & -0.95 \\
\hline \hline
\end{tabular}

solution for uniform flow Mach numbers of 0.0 and 0.257 . The real and imaginary part of the least attenuated axial wavenumber (i.e., $K_{0}$ ) and the corresponding mode eigenvalue (i.e., $\lambda_{0}$ ) for the assumed impedance spectra were calculated from Eqs. (15) and (16). It should be noted that the Mach number of 0.257 is used because it represents the average Mach number in the GFIT when the targeted centerline Mach number is set at 0.3 . The least attenuated mode coefficient, $A_{0}$, is chosen to correspond to a $120 \mathrm{~dB}$ sound pressure level. Recall that the LEE eduction method requires three pieces of information to educe the impedance, 1$)$ the inflow acoustic pressure $(p(0, L))$ and acoustic particle velocity $(v(0, x)), 2)$ the outflow acoustic pressure $(p(L, x))$, and 3$)$ the acoustic pressure at the seventy-nine microphone locations $\left(p\left(z_{J}, x_{J}\right), J=1,2,3, \ldots 79\right)$. This information is obtained from the least attenuated mode solution given in Eq. (14).

The educed normalized resistance and reactance of the liner predicted from the CHE and LEE methods, using synthesized data and with the flow off (i.e., $M_{0}=0.0$ ) is shown in Fig. 5. The closed symbols (labeled Res) are the educed normalized resistance spectra and the open symbols (labeled Rea) are the educed normalized reactance spectra. The curve labeled "LEE" is educed from the linearized Euler equations, the curve marked "CHE" is educed from the Convected Helmholtz equation, and the curve marked "EXP" represents the values from Table 1. LEE and CHE educed impedance are well correlated with each other and are not distinguishable from the values in Table 1. Results similar to those of Fig. 5 but with flow $\left(M_{0}=0.257\right)$ are shown in Fig. 6. Again, it is observed that the LEE and CHE educed impedances are well correlated with each other and are not distinguishable from the values in Table 1 for this flow condition. These results confirm that the LEE method is an effective tool for educing impedance when the input data is void of measurement uncertainties and boundary layer effects.

In the second problem, the LEE method is tested with measured data acquired in the GFIT. The measured GFIT data contains measurement uncertainties and realistic boundary layer effects. These characteristics of the measured data are expected to affect the quality of educed impedance. The primary objective of this second problem is to determine if the LEE and CHE educed impedances are comparable when measured data are used. Impedances educed using the LEE method are compared to those predicted from the CHE method when the sound propagates with the flow (i.e., the sound source is located upstream of the liner) and when the sound propagates against the flow (i.e., the sound source is located downstream of the liner). Figure 7 shows a plot of the educed normalized resistance for the conventional liner without flow (i.e., $M_{0}=0.0$ ). Results are shown for both the CHE and the LEE methods and for an upstream source (see the curve labeled "U") and a downstream source (see the curve labeled "D"). Although the impedance of the liner is not known a priori, it is expected that the educed impedances for the upstream and downstream sources will match (i.e., the impedance of the liner is a property of the acoustic material and not of the direction of sound propagation). In the absence of flow and with measured data, the normalized resistance educed from the LEE and the CHE methods are well matched to each other for both the upstream and downstream source for the conventional liner (see Fig. 7), except at 0.4 and $0.6 \mathrm{kHz}$. These are frequencies in the spectrum where the attenuation 


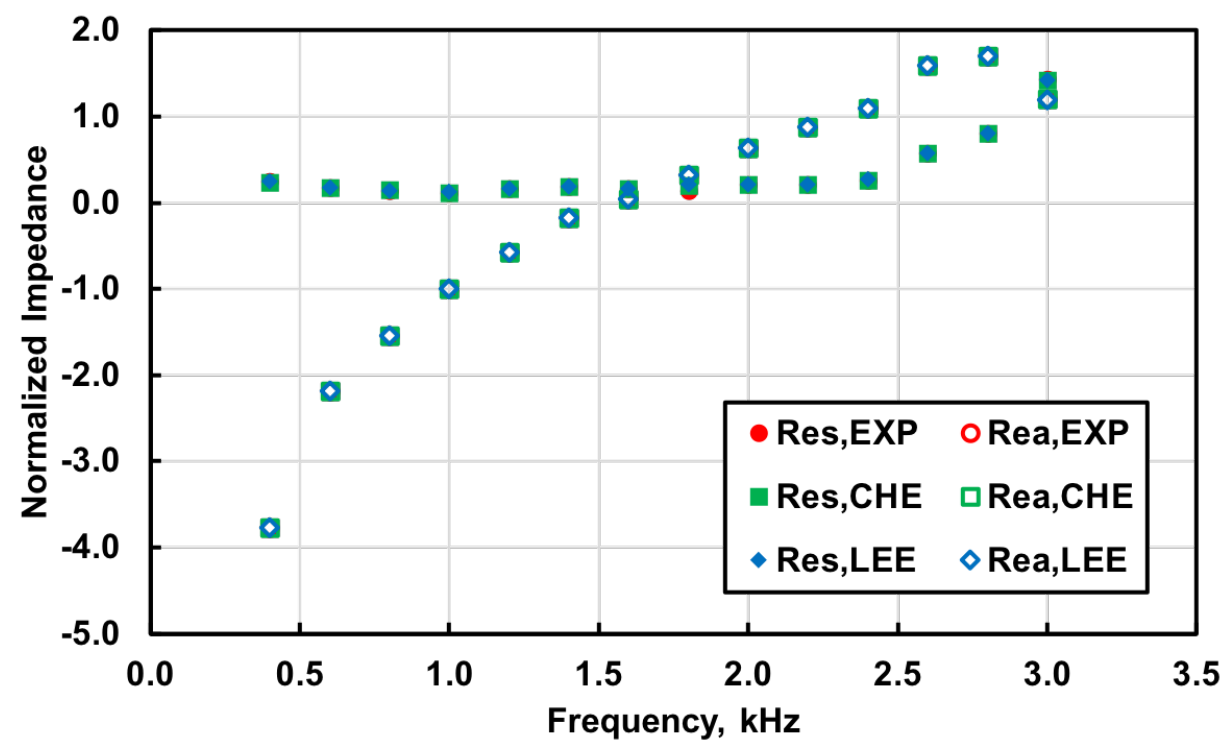

Figure 5. Comparison of educed normalized resistance and reactance at Mach 0.0 with synthesized data.

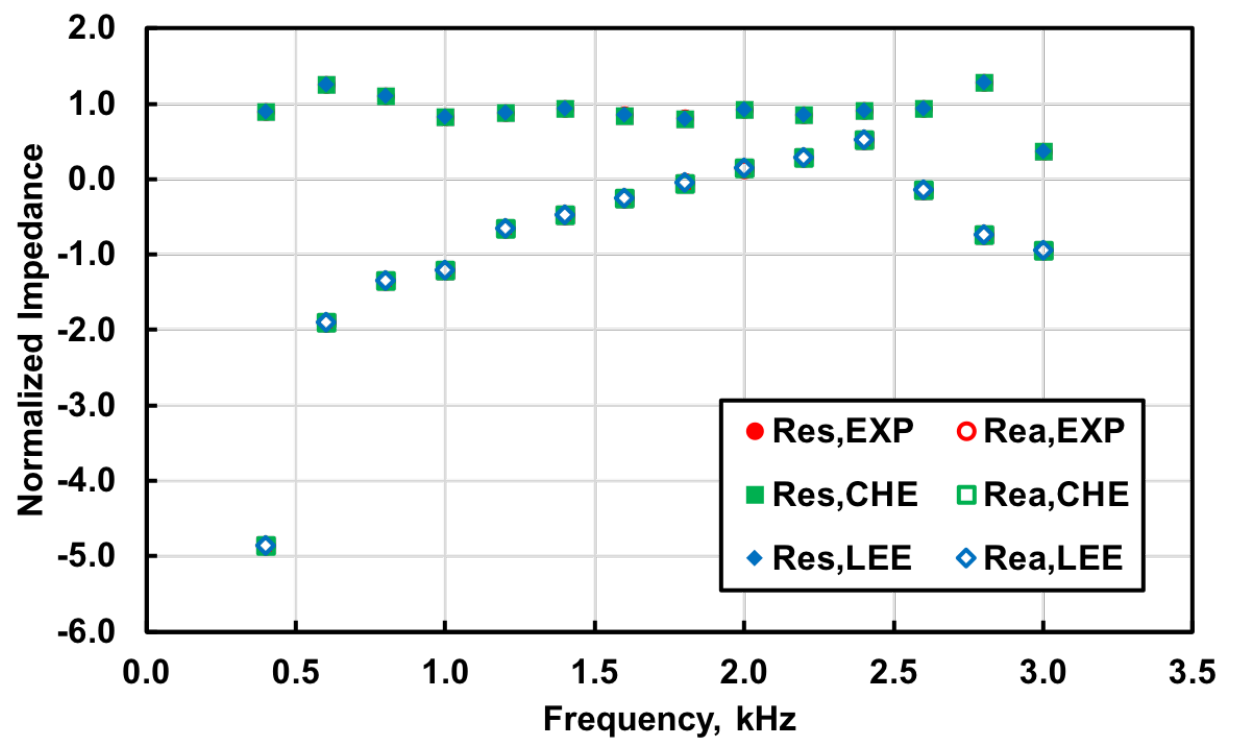

Figure 6. Comparison of educed normalized resistance and reactance at Mach 0.257 with synthesized data.

produced by the liner is extremely low. Surprisingly, the resistance educed for the upstream and downstream source is perfectly matched at $0.4 \mathrm{kHz}$ with the LEE method. However, the CHE method does not exhibit this trend at 0.4 $\mathrm{kHz}$. Observe also that at $0.6 \mathrm{kHz}$, the resistance educed with the downstream source is identical for the CHE and LEE eduction methods. However, there is considerable scatter in the educed resistance (between the CHE and LEE method) for the upstream source at $0.6 \mathrm{kHz}$.

Figure 8 gives the educed normalized reactance for the conventional liner in the absence of flow but using the measured data. The educed reactances for the CHE and LEE methods are well matched to each other across the frequency range. Further, the normalized reactance predicted from both the upstream and downstream sources are observed to be in very good agreement with one another using either the LEE or the CHE.

Figure 9 compares the CHE and LEE educed normalized resistances for the conventional liner at Mach 0.257 


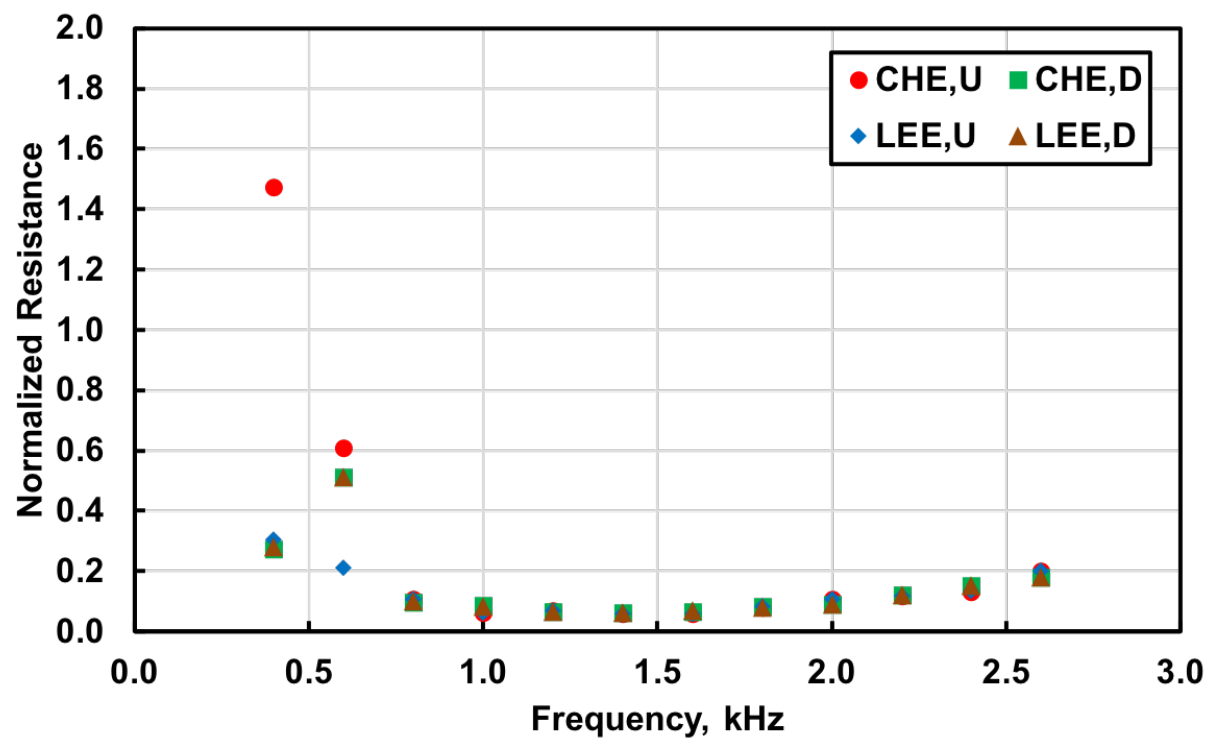

Figure 7. Comparison of educed normalized resistance at Mach 0.0 using measured data.

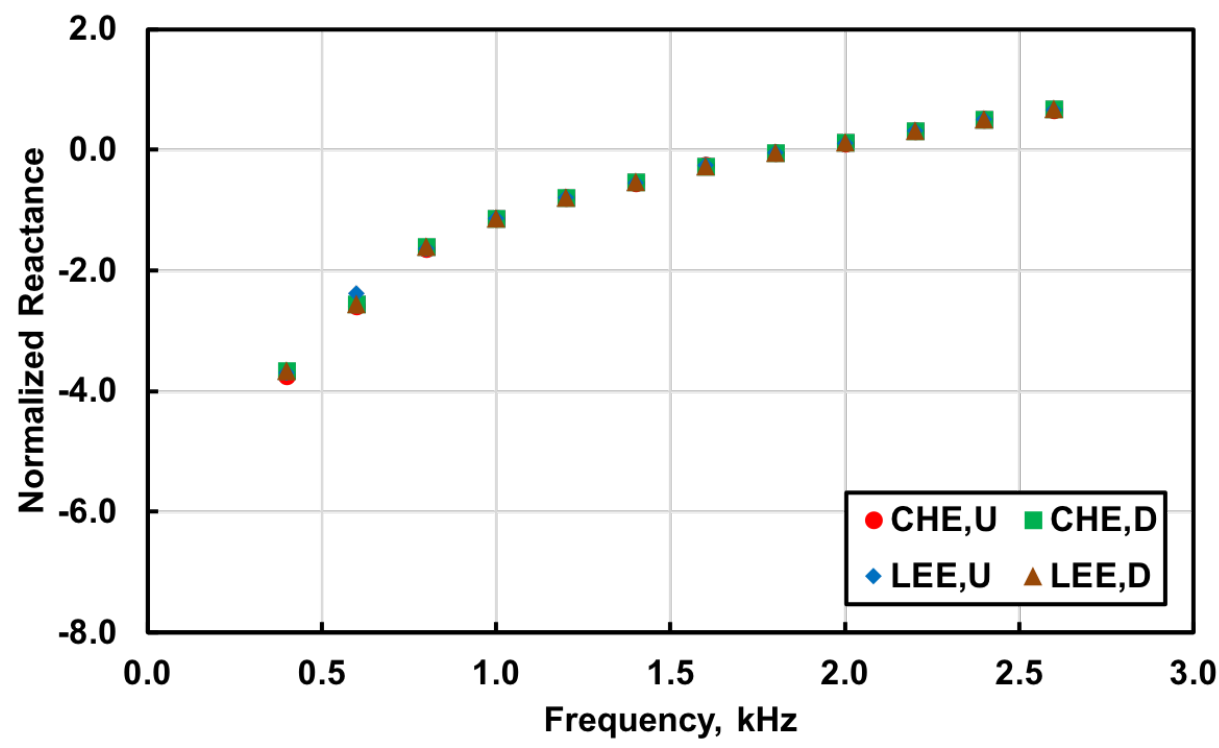

Figure 8. Comparison of educed normalized reactance at Mach 0.0 using measured data.

using measured data. In general, the results for the CHE and LEE compare favorably. However, the resistances for the upstream source are distinctly different from those attained with the downstream source. The differences are significant for frequencies below about $1000 \mathrm{~Hz}$. The corresponding reactance spectra are depicted in Fig. 10. Again, the results for the CHE and LEE are well matched, but the results for the upstream and downstream sources show clear differences, albeit much less than is present in the resistance results. The results presented in Figs. 9 and 10 demonstrate that differences in educed impedances achieved with upstream and downstream sources are not eliminated by this change in the model (i.e., from the CHE model to the current LEE model).

As mentioned earlier, it is generally accepted that the impedance of an acoustic liner is an intrinsic parameter, and should therefore be independent of the location of the source (or the direction of mean flow). At least two hypotheses have been considered to explain these apparent differences. Schulz et al. ${ }^{16}$ suggest that the effects of 


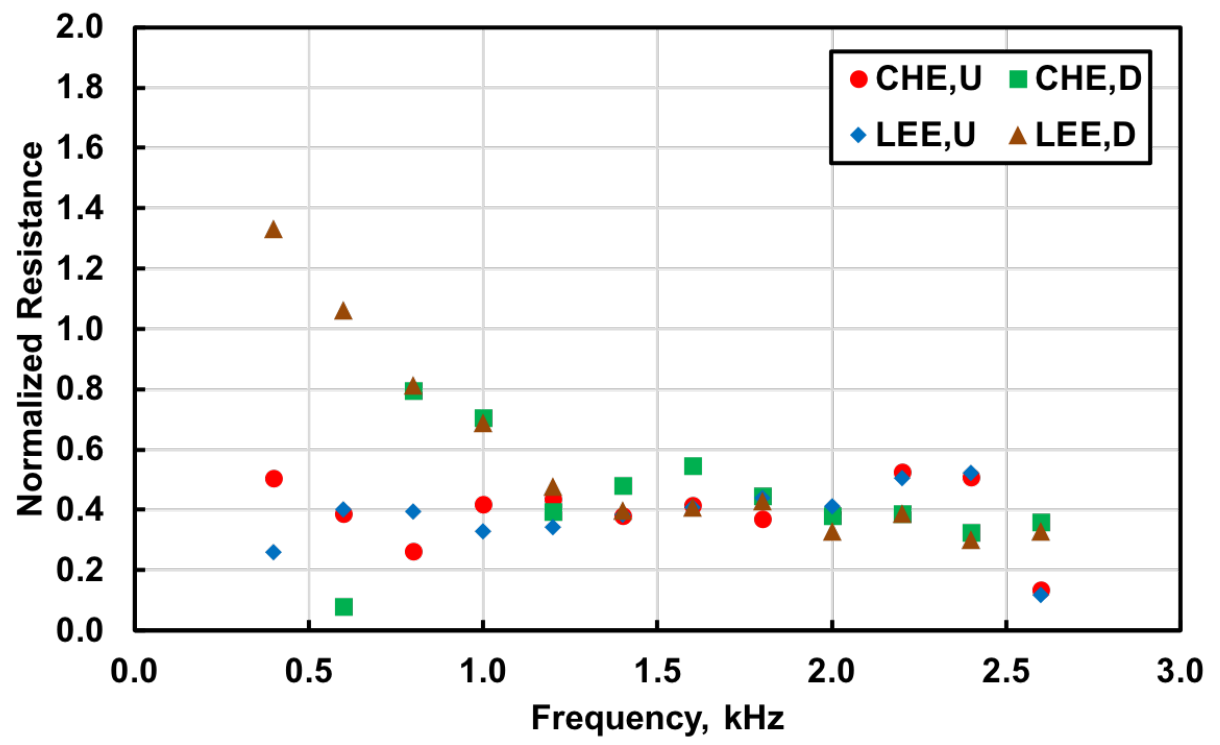

Figure 9. Comparison of educed normalized resistance at Mach 0.257 using measured data.

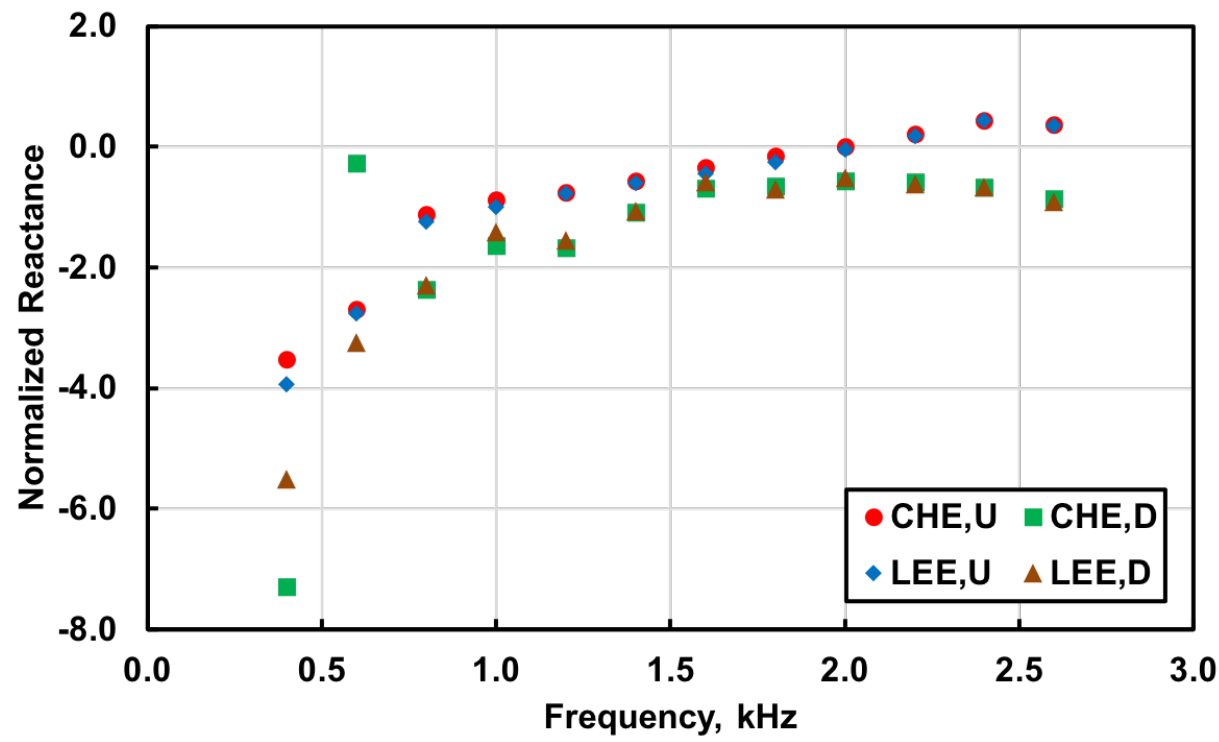

Figure 10. Comparison of educed normalized reactance at Mach 0.257 using measured data.

a momentum transfer impedance must be included to account for differences encountered with the upstream and downstream sources. A second hypothesis is discussed in a companion pape ${ }^{17}$ whereby it is demonstrated that a very small uncertainty in the uniform flow Mach number can account for the differences in the respective results.

\section{B. Impedance Eduction in the Presence of Finite Boundary Layer Thickness and Test Data}

In this section, the LEE method of impedance eduction is tested under the assumption of uniform mean flow (i.e., $M_{0}(z, x)=$ constant $)$ and finite boundary layer thickness. Recall that the CHE method uses the Ingard-Myers boundary condition and cannot account for the effects of finite boundary layer thickness. Figure 11 compares the educed normalized resistance at Mach 0.257 as a function of the boundary layer thickness for the upstream source. The boundary 


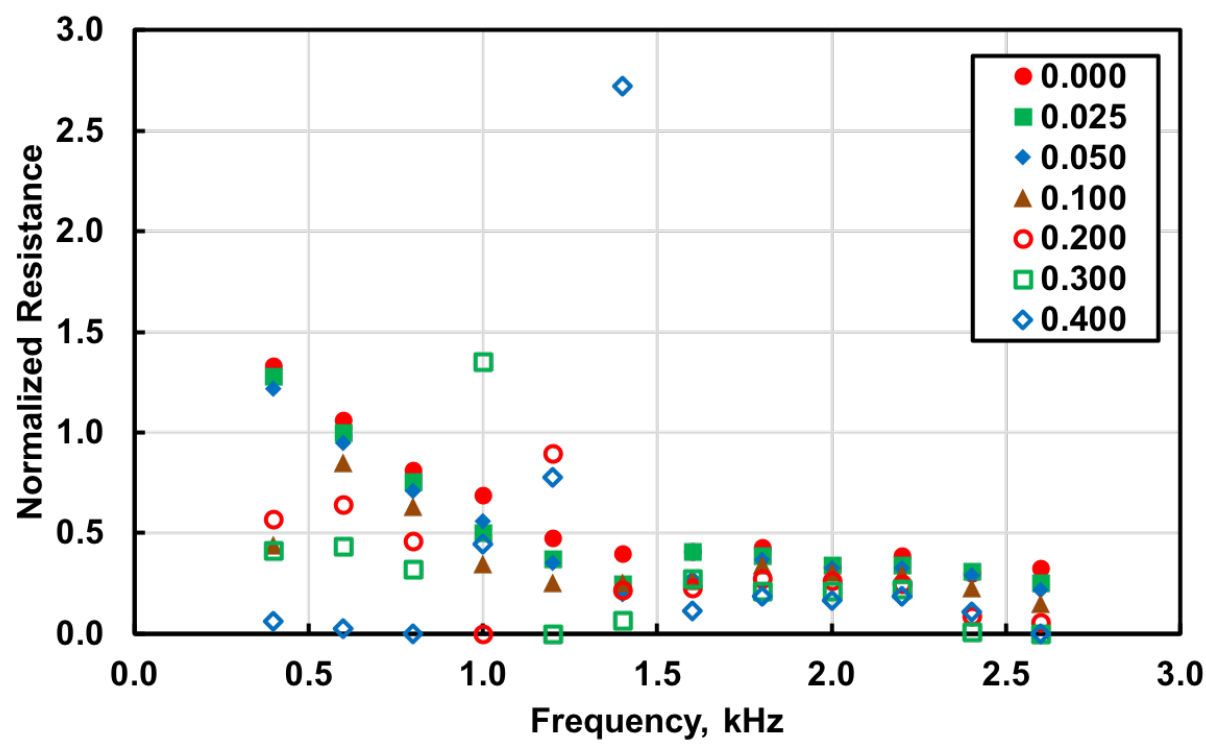

Figure 11. Effects of boundary layer thickness on the educed normalized resistance at Mach 0.257 using measured data.

layer thickness, $\sigma$, ranges from 0.0 to 0.4 inches in the figure. The impact of the boundary layer thickness on the normalized resistance occurs for the most part at source frequencies less than $1.5 \mathrm{kHz}$. It is observed that the educed normalized resistance is reduced with increasing boundary layer thickness. Figure 12 compares the educed normal-

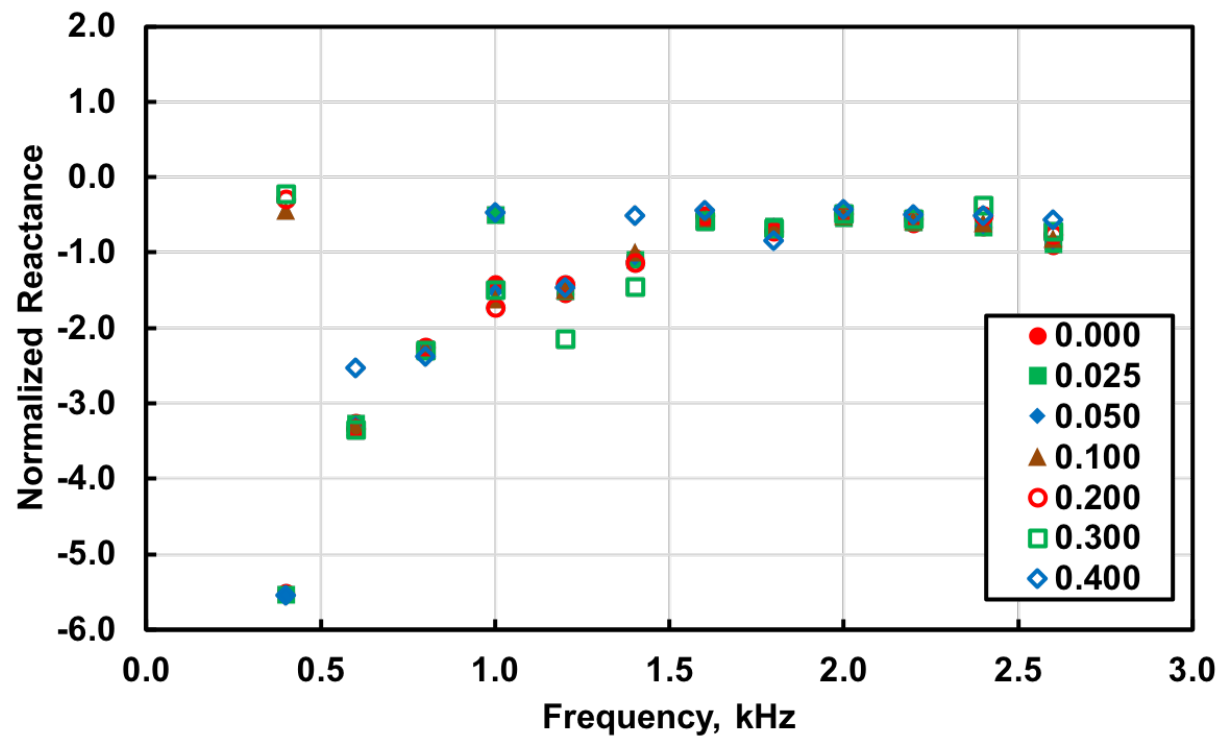

Figure 12. Effects of boundary layer thickness on the educed normalized reactance at Mach 0.257 using measured data.

ized reactance at Mach 0.257 as a function of the boundary layer thickness for the upstream source. The impact of the boundary layer thickness on the normalized reactance is observed to be small except in the low frequency region where the attenuation is low. Similar trends to those shown in Fig 11 and Fig. 12 were observed for the downstream source so these results are not presented for the sake of brevity. 


\section{Conclusions}

A LEE method of impedance eduction has been presented. The method allows for mean flow gradients in both coordinate directions and variable impedance liners. However, only uniform values of these parameters are evaluated in this paper. Based upon the results of this paper, the following conclusion are drawn:

1. When the impedance eduction data is synthesized from the exact solution, the CHE and LEE methods educe the same impedance.

2. When used with test data, the CHE and LEE methods educe identical impedances in the absence of mean flow and the educed impedance for an upstream and a downstream source are well matched.

3. When the flow is turned on and measured data is used, the CHE and LEE methods educe the same impedance for an upstream source. The corresponding results educed with a downstream source also compare favorably. However, there are significant differences between the educed impedances for the upstream and downstream sources. This result confirms that the differences in impedances educed with the upstream and downstream sources are not due to the choice of impedance eduction model.

4. The boundary layer thickness has little impact on the educed normalized reactance. The primary effect of the boundary layer thickness is to reduce the normalized resistance. This effect is most dramatic in the mid to low frequency range and for thick boundary layers.

\section{Acknowledgments}

This work was funded by the Advanced Air Transport Technology Project of the NASA Advanced Air Vehicles Program.

\section{References}

\footnotetext{
${ }^{1}$ Jing, X., Peng, S., and Sun, X., "A Straightforward Method for Wall Impedance Eduction in a Flow Duct," Journal of the Acoustical Society of America, Vol. 124, No. 1, July 2008, pp. 227-234.

${ }^{2}$ Renou, Y. and Aurégan Y., "Failure of the Ingard-Myers Boundary Condition for a Lined Duct: An Experimental Investigation," Journal of the Acoustical Society of America, Vol. 130, No. 1, July 2011, pp. 52-60.

${ }^{3}$ Watson, W. R., Carpenter, M. H., and Jones, M. G., "Performance of Kumaresan and Tufts Algorithm in Liner Impedance Eduction with Flow," AIAA Journal, Vol. 53, No. 4, Feb. 2015, pp. 1091-1102.

${ }^{4}$ Weng, C., Schulz, A., Ronneberger, D., and Bake, F., "Impedance Eduction in the Presence of Turbulent Shear Flow Using the Linearized Navier-Stokes Equations," AIAA Paper 2017-3182, June 2017.

${ }^{5}$ Watson, W. R., Jones, M. G., and Parrott, T. L., "Validation of an Impedance Eduction Method in Flow," AIAA Journal, Vol. 37, No. 7, July 1999, pp. 818-824.

${ }^{6}$ Jones, M. G., Watson, W. R., Tracy, M. B., and Parrott, T. L., "Comparison of Two Waveguide Methods for Educing Liner Impedance in Grazing Flow," AIAA Journal, Vol. 42, No. 2, Feb. 2004, pp. 232-240.

${ }^{7}$ Jones, M., Watson, W., and Parrott, T., "Benchmark Data for Evaluation of Aeroacoustic Propagation Codes with Grazing Flow," AIAA Paper 2005-2853, May 2005.

${ }^{8}$ Watson, W. R., Jones, M. G., and Parrott, T. L., "Comparison of a Convected Helmholtz and Euler Model for Impedance Eduction in Flow," AIAA Paper 2006-2643, May 2006.

9Jones, M. G., Watson, W. R., and Nark, D. M., "Effects of Flow Profile on Educed Acoustic Liner Impedance," AIAA Paper 2010-3763, June 2010

${ }^{10}$ Watson, W. R., and Jones, M. G., "A Comparative Study of Four Impedance Eduction Methodologies Using Several Test Liners," AIAA Paper 2013-2274, June 2013.

${ }^{11}$ Myers, M. K., “On the Acoustic Boundary Condition in The Presence of Flow,” Journal Of Sound And Vibrations, Vol. 71, No. 3, pp. 429-434, Aug. 1980.

${ }^{12}$ Rienstra, S. W. and Darau, M., “ Mean Flow Boundary Layer Effects of Hydrodynamic Instability of Impedance Wall,” Procedia Engineering, Vol. 6, 2010, pp. 124-132.

${ }^{13}$ Anderson, D., Tannehill, J. , and Pletcher, R., Computational Fluid Mechanics and Heat Transfer, Hemisphere Publishing Corporation, New York, 1984, pp. 144-145.

${ }^{14}$ Desia, C., and Abel, John F., Introduction to the Finite Element Method, Van Nostrand Reinhold, New York, 1972, pp. 67-214.

${ }^{15}$ Stewart, G. W. III, “A Modification of Davidon's Minimization Method to Accept Difference Approximations of Derivatives," Journal of ACM, Vol. 14, No. 1, 1967, pp 72-83.

${ }^{16}$ Schultz, A., Weng, C., Bake, F., and Enghardt, L., "Modeling of Liner Impedance with Grazing Shear Flow Using a New Momentum Transfer Boundary Condition," AIAA Paper 2017-3377, June 2017.

${ }^{17}$ Nark, D., Jones, M., and Piot, E., “ Assessment of Axial Wave Number and Mean Flow Uncertainty on Acoustic Liner Impedance Eduction,” AIAA Paper accepted for presentation at Aviation 2018, Atlanta, Georgia.
} 
${ }^{18}$ Jones, E., Oliphant, T., and Peterson, P., “Open Source Scientific Tools for Python,” URL http//www.scipy.org/, 2001.

15 of 15

American Institute of Aeronautics and Astronautics 